\title{
Increased liver stiffness in patients with severe sleep apnoea and metabolic comorbidities
}

\author{
Wojciech Trzepizur ${ }^{1,2}$, Jérôme Boursier ${ }^{3,4}$, Marc Le Vaillant ${ }^{5}$, \\ Pierre-Henri Ducluzeau ${ }^{6}$, Séverine Dubois $^{2,7}$, Samir Henni ${ }^{8,9}$, \\ Pierre Abraham ${ }^{8,9}$, Christophe Aubé ${ }^{4,10}$, Paul Calès ${ }^{3,4}$ and \\ Frédéric Gagnadoux ${ }^{1,2}$ on the behalf of the METABOL group ${ }^{11}$
}

Affiliations: 'Dépt de Pneumologie, Centre Hospitalier Universitaire, Angers, France. ${ }^{2}$ INSERM UMR 1063 , Université d'Angers, Angers, France. ${ }^{3}$ Dépt d'Hépato-Gastroentérologie, Centre Hospitalier Universitaire, Angers, France. ${ }^{4}$ Laboratoire HIFIH, EA3859, Université d'Angers, Angers, France. ${ }^{5}$ Institut de Recherche en Santé Respiratoire des Pays de la Loire, Beaucouzé, France. 'Unité d’Endocrinologie-Diabétologie-Nutrition, Pole de Médecine, Centre Hospitalier Universitaire, Tours, France. ${ }^{7}$ Dépt d'Endocrinologie, Diabétologie et Nutrition, Centre Hospitalier Universitaire, Angers, France. ${ }^{8}$ Dépt de Médecine du Sport et Explorations Fonctionnelles Vasculaires, Centre Hospitalier Universitaire, Angers, France. 9 Institut MITOVASC, UMR CNRS 6015, INSERM 1083, Université d'Angers, Angers, France. ${ }^{10}$ Dépt de Radiologie, Centre Hospitalier Universitaire, Angers, France. ${ }^{11} \mathrm{~A}$ full list of members of the METABOL group can be found in the Acknowledgements section.

Correspondence: Wojciech Trzepizur, Dépt de Pneumologie, Centre Hospitalier Universitaire, 4 rue Larrey, 49100 Angers, France. E-mail: wotrzepizurdachu-angers.fr

@ERSpublications

In patients with metabolic comorbidities, severe OSA is independently linked with an increased risk of liver disease, as assessed by liver stiffness measurement http://ow.ly/e94330jWAZr

Cite this article as: Trzepizur W, Boursier J, Le Vaillant M, et al. Increased liver stiffness in patients with severe sleep apnoea and metabolic comorbidities. Eur Respir J 2018; 51: 1800601 [https://doi.org/10.1183/ 13993003.00601-2018].

ABSTRACT The goal of this study was to assess the relationship between the severity of obstructive sleep apnoea (OSA) and liver stiffness measurement (LSM), one of the most accurate noninvasive screening tools for liver fibrosis in nonalcoholic fatty liver disease.

The study included 147 patients with at least one criterion for the metabolic syndrome, assessed by polysomnography for suspected OSA. LSM was performed using transient elastography (FibroScan). Significant liver disease and advanced liver fibrosis were defined as LSM $\geqslant 7.3$ and $\geqslant 9.6 \mathrm{kPa}$, respectively.

23 patients were excluded because of unreliable LSM. Among 124 patients, 34 (27.4\%) had mild OSA, $38(30.6 \%)$ had moderate OSA and $52(42.0 \%)$ had severe OSA. LSM values were $7.3-<9.6 \mathrm{kPa}$ in 18 (14.5\%) patients and $\geqslant 9.6 \mathrm{kPa}$ in $15(12.1 \%)$ patients. A dose-response relationship was observed between OSA severity and LSM values $(\mathrm{p}=0.004)$. After adjustment for age, sex, metabolic syndrome and insulin resistance, severe OSA was associated with an increased risk of LSM $\geqslant 7.3 \mathrm{kPa}$ (OR 7.17, 95\% CI 2.5120.50 ) and $\mathrm{LSM} \geqslant 9.6 \mathrm{kPa}$ (OR 4.73, 95\% CI 1.25-17.88).

In patients with metabolic comorbidities, severe OSA is independently associated with increased liver stiffness, which may predispose to a higher risk of significant liver disease and poorer prognosis. 


\section{Introduction}

The growing worldwide obesity epidemic is resulting in an increased prevalence of obesity-related chronic diseases, including metabolic syndrome, type 2 diabetes, obstructive sleep apnoea (OSA) and nonalcoholic fatty liver disease (NAFLD) [1,2]. The risk factors, pathophysiology and consequences of these chronic diseases may share common pathways and could impact on each other. Experimental and clinical data have suggested that OSA and its hypoxia-related consequences may contribute to the development and exacerbation of NAFLD, the hepatic manifestation of obesity and metabolic syndrome [3]. In morbidly obese patients undergoing bariatric surgery during which a liver needle biopsy was obtained, a doseresponse relationship has been demonstrated between intermittent hypoxia severity and the severity of liver injury [4].

NAFLD includes a wide spectrum of histological lesions, ranging from simple steatosis, steatohepatitis and fibrosis, potentially leading to end-stage cirrhosis. Convergent findings from longitudinal studies indicate that the amount of liver fibrosis is the main determinant of the liver-related prognosis in patients with NAFLD [5]. The reference procedure for liver fibrosis evaluation is histological examination of a liver biopsy. However, liver biopsy is an invasive procedure, and is associated with considerable cost and a significant complication rate of $\sim 1 \%$ [6]. Most importantly, in view of the magnitude of the current obesity epidemic and the subsequent number of patients at risk for liver alterations, liver biopsy cannot be considered to be a practical, efficient and large-scale tool to identify patients at risk of advanced fibrosis. Noninvasive screening tools for liver fibrosis, including blood tests and transient elastography devices, have recently been developed and validated in at-risk populations $[7,8]$. Transient elastography is a recognised rapid and user-friendly technique that can be easily performed at the bedside or in the outpatient clinic with immediate results and good reproducibility [9]. Liver stiffness measurement (LSM) by transient elastography (FibroScan; Echosens, Paris, France) has been demonstrated to be one of the most accurate tests for the noninvasive diagnosis of liver fibrosis in NAFLD [10]. LSM has also been found to be a reliable prognostic marker for the stratification of NAFLD patients into several subgroups with significantly different prognosis [10]. The aim of this cross-sectional cohort study was to determine whether severe OSA is associated with significant liver disease evaluated by LSM.

\section{Patients and methods}

Patients

This study was nested within the NUMEVOX cohort (ClinicalTrials.gov identifier NCT00997165) conducted at Angers University Hospital (Angers, France). The primary objective of the NUMEVOX cohort was to evaluate the impact of fat distribution on vascular and metabolic progression in patients with at least one criterion for metabolic syndrome referred to Angers University Hospital [11, 12]. Exclusion criteria were age $<18$ or $>65$ years, cause of chronic liver disease other than NAFLD, complicated cirrhosis and insulin-treated diabetes. All patients included in the NUMEVOX cohort were assessed by the Berlin questionnaire to evaluate the clinical likelihood of OSA [13]. Patients subsequently investigated by overnight polysomnography (PSG) based on a positive Berlin questionnaire were included in the present study. The study was approved by the institutional ethics committee, and oral and written consent was obtained from each patient by means of a process validated by the Angers University Hospital Research Ethics Committee (CPP OUEST II, ANGERS).

\section{Clinical assessment and blood tests}

Baseline evaluation included anthropometric data, medical history and medication use. Patients receiving antidiabetic treatment were considered to have diabetes. Hypertension was defined as a physician's diagnosis combined with treatment with appropriate medication. Waist circumference (in centimetres) was measured using a nonelastic measuring tape. Excessive daytime sleepiness was evaluated by the Epworth Sleepiness Scale [14]. All patients underwent fasting blood tests, including blood glucose, insulin, liver enzymes (alanine aminotransferase (ALT), aspartate aminotransferase (AST), $\gamma$-glutamyl transpeptidase (GGT) and alkaline phosphatase) and total bilirubin. The homeostasis model assessment for insulin resistance (HOMA-IR) index was calculated from fasting glucose and insulin concentrations as: insulin $\left(\mathrm{mIU} \cdot \mathrm{L}^{-1}\right) \times$ glucose $\left(\mathrm{mmol} \cdot \mathrm{L}^{-1}\right) / 22.5$ [15]. The presence of metabolic syndrome was defined according to the National Cholesterol Education Program Adult Treatment Panel III clinical criteria [11].

\section{Liver stiffness measurement}

LSM was performed with the FibroScan device under fasting conditions according to the manufacturer's recommendations [9] by a specialised nurse blinded to the patient's data. The examination was conducted using the standard $\mathrm{M}$ probe. Ten valid acquisitions were recorded and the result (in kilopascals) was expressed as the median of these valid acquisitions. Based on the meta-analysis published by TsochATzIS et al. [16], an LSM cut-off value $\geqslant 7.3 \mathrm{kPa}$ was used to identify patients with significant liver disease. 
Advanced liver fibrosis was defined by the classical LSM cut-off value $\geqslant 9.6 \mathrm{kPa}$ used in NAFLD [17, 18]. According to previously validated reliability criteria for LSM with FibroScan, patients with LSM $\geqslant 7.3 \mathrm{kPa}$ and interquartile range/median ratio $>0.30$ were considered to be poorly reliable and were excluded from the analysis $[19,20]$.

\section{Polysomnography}

As previously described [21], overnight in-laboratory PSG (CID 102; Cidelec, Angers, France) was performed (within 1 month of LSM) with continuous recording of the following channels: electroencephalogram, electro-oculogram, chin electromyogram, arterial oxygen saturation, nasal-oral airflow, ECG, chest and abdominal wall motion, and body position. Respiratory events were scored manually according to standard criteria [22]. Apnoea was defined as complete cessation of airflow and hypopnoea was defined as an at least $30 \%$ decrease in the nasal pressure signal combined with either $\geqslant 3 \%$ arterial oxygen desaturation or an arousal, both lasting at least $10 \mathrm{~s}$. The apnoea-hypopnoea index (AHI) and $3 \%$ oxygen desaturation index (ODI) were defined by the number of events per hour of sleep. The following commonly used cut-offs for AHI were used to define categories of OSA severity: $5-<15$ events $\cdot \mathrm{h}^{-1}$ for mild OSA, $15-<30$ events. $\mathrm{h}^{-1}$ for moderate OSA and $\geqslant 30$ events $\cdot \mathrm{h}^{-1}$ for severe OSA. The percentage of sleep time with oxygen saturation $<90 \%$ (T90) was also calculated.

\section{Statistical analysis}

All statistical analyses were performed with SAS/STAT package 2002-2003 (SAS Institute, Cary, NC, USA). Results were expressed as percentage, mean with standard deviation and odds ratio with $95 \%$ confidence interval. A two-tailed p-value $<0.05$ was considered significant. The primary dependent variables of interest were the presence of significant liver disease and advanced liver fibrosis as defined by LSM $\geqslant 7.3$ and $\geqslant 9.6 \mathrm{kPa}$, respectively. The primary independent variable was the severity of OSA as assessed by AHI and two different indices of nocturnal hypoxaemia (ODI and T90). Patient characteristics across categories of OSA severity were compared using a Chi-squared test for categorical variables and a two-sample t-test for continuous variables. Logistic regression procedures were performed to calculate the adjusted odds ratio with $95 \%$ confidence interval for having LSM $\geqslant 7.3$ or $\geqslant 9.6 \mathrm{kPa}$ according to the severity of OSA and nocturnal hypoxaemia. To adjust for potential confounders, the following covariates likely to interfere with NAFLD were entered in the multivariate analysis: age, sex, waist circumference, diabetes, hypertension, metabolic syndrome and HOMA-IR.

\section{Results}

Of the 414 patients included in the NUMEVOX cohort between March 2008 and August 2017, 147 patients had a positive Berlin questionnaire and were further investigated by overnight PSG. 23 of these patients were excluded from the analysis due to unreliable transient elastography with LSM $\geqslant 7.3 \mathrm{kPa}$ and interquartile range/median ratio $>0.30$. The final study sample size therefore comprised 124 patients (figure 1). OSA was confirmed by PSG in all patients, including 34 (27.4\%) with mild OSA, 38 (30.6\%) with moderate OSA and $52(42.0 \%)$ with severe OSA. As shown in table 1, the study population consisted of typical OSA patients, predominantly male, obese or overweight, frequently presenting metabolic comorbidities. As expected, increasing OSA severity was associated with higher body mass index (BMI) $(\mathrm{p}=0.06)$, waist circumference $(\mathrm{p}=0.02)$, ODI $(\mathrm{p}<0.0001), T 90(\mathrm{p}=0.002)$ and higher proportions of male patients $(\mathrm{p}=0.02)$ with metabolic syndrome $(\mathrm{p}=0.02)$. Increasing OSA severity was also associated with higher LSM values $(\mathrm{p}=0.004)$ with a marked increase between mild-to-moderate OSA and severe OSA.

Among the 124 patients with mild-to-severe OSA, 91 (73.4\%) had LSM <7.3 kPa, 18 (14.5\%) had LSM $7.3-<9.6 \mathrm{kPa}$ indicating significant liver disease and $15(12.1 \%)$ had LSM $\geqslant 9.6 \mathrm{kPa}$ indicating advanced liver fibrosis. As shown in table 2, patients with elevated LSM values had significantly higher levels of liver enzymes (ALT, $p=0.01$; AST, $p=0.0003$; GGT, $p=0.01$ ) and total bilirubin $(\mathrm{p}=0.002)$. HOMA-IR increased with elevated LSM values, but this increase was not significant $(\mathrm{p}=0.39)$.

As shown in figure 2, a dose-response relationship was observed between OSA severity and LSM values, with a marked increase in the proportion of patients with significant liver disease (LSM $7.3-<9.6 \mathrm{kPa}$ ) and advanced liver fibrosis (LSM $\geqslant 9.6 \mathrm{kPa}$ ) between mild-to-moderate and severe OSA. For subsequent analysis, the AHI variable was dichotomised into two categories (i.e. AHI $\geqslant 30$ events. $\mathrm{h}^{-1}$ (severe OSA) versus AHI $<30$ events. $\mathrm{h}^{-1}$ (mild-to-moderate OSA)). The study sample was also dichotomised into two categories for physiological indices of nocturnal hypoxaemia according to the median value of the variable.

Unadjusted and adjusted odds ratios for LSM $\geqslant 7.3 \mathrm{kPa}$ according to OSA severity are presented in table 3 . On univariate analysis, severe OSA (AHI $\geqslant 30$ events.h ${ }^{-1}$ ) was associated with a significantly increased risk of LSM $\geqslant 7.3 \mathrm{kPa}$ (OR 5.66, 95\% CI 2.32-13.79). The association between OSA and significant liver disease remained highly significant after adjustment for age, sex, waist circumference, hypertension and diabetes 
414 patients presenting with at least one criterion for the metabolic syndrome were included in the NUMEVOX cohort between March 2008 and August 2017

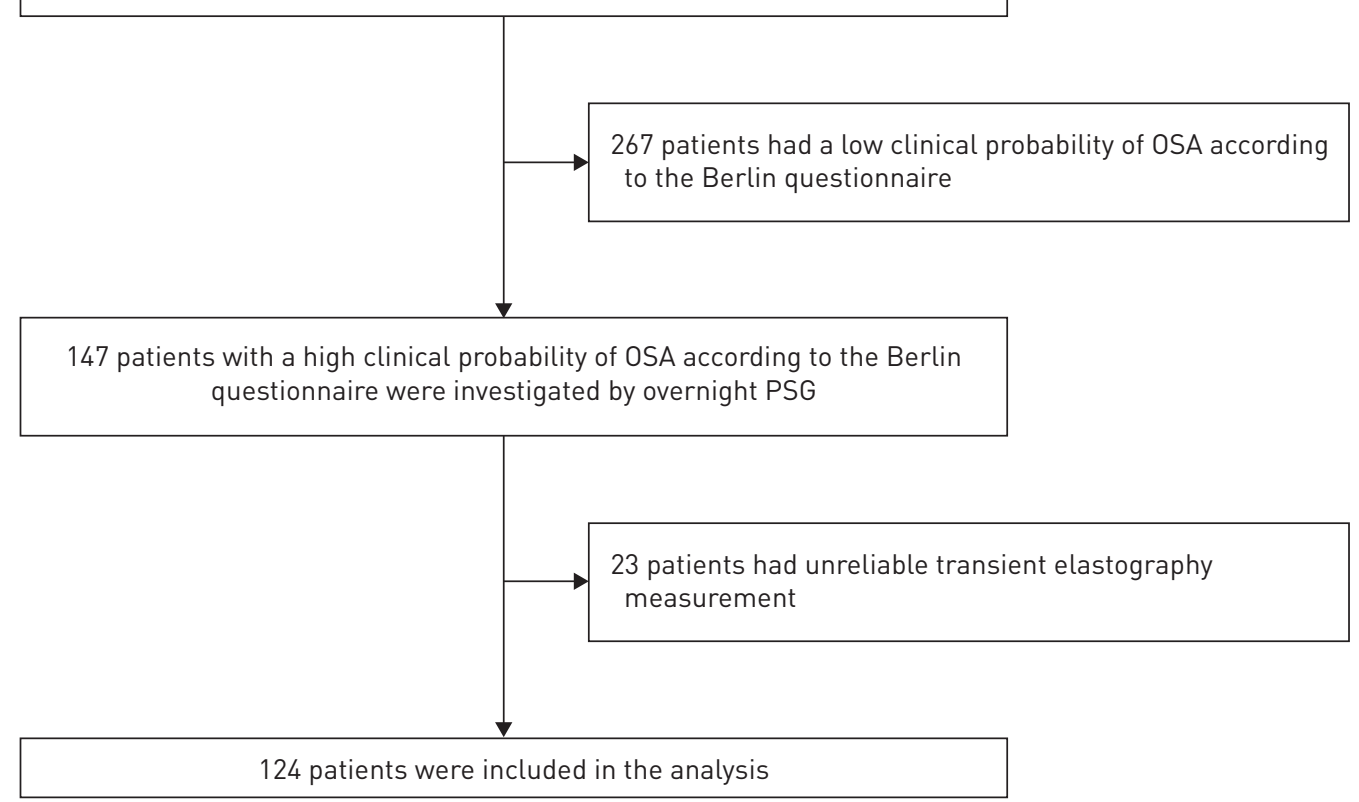

FIGURE 1 Flow diagram of subjects during the study. OSA: obstructive sleep apnoea; PSG: polysomnography.

(model 1: OR 6.59, 95\% CI 2.35-18.49), adjustment for age, sex and metabolic syndrome (model 2: OR 5.63, 95\% CI 2.17-14.63) or adjustment for age, sex, metabolic syndrome and HOMA-IR (model 3: OR 7.17, 95\% CI 2.51-20.50).

The associations between two different indices of nocturnal hypoxaemia and LSM $\geqslant 7.3 \mathrm{kPa}$ were also investigated. As shown in table 3, increasing hypoxaemia during sleep, as reflected by ODI and T90, was significantly associated with an increased risk of significant liver disease. On multivariate analysis, the association remained significant for ODI and T90 according to models 1, 2 and 3.

A subsequent analysis calculated the unadjusted and adjusted odds ratios for LSM $\geqslant 9.6 \mathrm{kPa}$ according to the severity of OSA and hypoxaemia during sleep. On multivariate analysis, severe OSA and elevated ODI $\left(\geqslant 19\right.$ events. $\mathrm{h}^{-1}$ of sleep) were associated with an increased risk of advanced liver fibrosis after adjustment

TABLE 1 Patient characteristics according to the severity of obstructive sleep apnoea (OSA)

\begin{tabular}{|c|c|c|c|c|c|}
\hline & All & Mild OSA & Moderate OSA & Severe OSA & p-value \\
\hline Subjects $\mathrm{n}$ & 124 & 34 & 38 & 52 & \\
\hline Female & 34.4 & 52.9 & 24.3 & 29.4 & 0.0247 \\
\hline Age years & $52.4 \pm 9.5$ & $49.7 \pm 10.8$ & $52.5 \pm 9.6$ & $53.7 \pm 7.9$ & 0.1513 \\
\hline BMI $\mathrm{kg} \cdot \mathrm{m}^{-2}$ & $29.9 \pm 4.8$ & $28.6 \pm 4.9$ & $29.6 \pm 4.2$ & $31.0 \pm 5.0$ & 0.0620 \\
\hline Waist circumference cm & $102.3 \pm 12.7$ & $97.4 \pm 14.7$ & $102.6 \pm 10.3$ & $105.2 \pm 12.1$ & 0.0195 \\
\hline Diabetes & 16.4 & 17.6 & 10.8 & 19.6 & 0.5313 \\
\hline Hypertension & 45.1 & 38.2 & 43.2 & 51.0 & 0.4939 \\
\hline Statin use & 26.2 & 20.6 & 16.2 & 37.3 & 0.0584 \\
\hline Metabolic syndrome & 52.5 & 32.4 & 59.5 & 60.8 & 0.0218 \\
\hline ESS score & $11.2 \pm 5.5$ & $10.4 \pm 5.2$ & $12.0 \pm 5.6$ & $11.1 \pm 5.6$ & 0.5984 \\
\hline AHI events $\cdot h^{-1}$ & $29.1 \pm 23.2$ & $6.6 \pm 3.7$ & $20.7 \pm 3.9$ & $50.2 \pm 20.7$ & $<0.0001$ \\
\hline ODI events: $\mathrm{h}^{-1}$ & $23.2 \pm 22.4$ & $7.4 \pm 16.3$ & $16.3 \pm 11.8$ & $38.7 \pm 22.1$ & $<0.0001$ \\
\hline T90 \% & $9.1 \pm 16.9$ & $2.1 \pm 7.4$ & $6.0 \pm 16.8$ & $16.1 \pm 19.1$ & 0.0002 \\
\hline LSM kPa & $6.6 \pm 4.3$ & $5.2 \pm 1.6$ & $5.8 \pm 2.3$ & $8.1 \pm 6.0$ & 0.0041 \\
\hline
\end{tabular}

Data are expressed as \% or mean \pm SD, unless otherwise stated. BMI: body mass index; ESS: Epworth Sleepiness Scale; AHI: apnoea-hypopnoea index; ODI: oxygen desaturation index; T90: sleep time with oxygen saturation $<90 \%$; LSM: liver stiffness measurement. 
TABLE 2 Biological data according to liver stiffness measurement (LSM)

\begin{tabular}{|c|c|c|c|c|}
\hline & \multicolumn{3}{|c|}{ LSM kPa } & \multirow[t]{2}{*}{ p-value } \\
\hline & $<7.3$ & $7.3-<9.6$ & $\geqslant 9.6$ & \\
\hline Subjects & 91 & 18 & 15 & \\
\hline Total bilirubin $\mu \mathrm{mol} \cdot \mathrm{L}^{-1}$ & $9.1 \pm 4.3$ & $10.0 \pm 4.8$ & $14.3 \pm 9.5$ & 0.0023 \\
\hline ALT IU. $L^{-1}$ & $39.7 \pm 24.5$ & $61.5 \pm 33.2$ & $58.6 \pm 33.6$ & 0.0014 \\
\hline AST IU:L-1 & $28.0 \pm 12.2$ & $42.6 \pm 27.5$ & $40.1 \pm 18.8$ & 0.0003 \\
\hline Alkaline phosphatase IU. $\mathrm{L}^{-1}$ & $69.3 \pm 23.7$ & $70.8 \pm 18.6$ & $72.2 \pm 25.4$ & 0.8924 \\
\hline GGT IU.L $\mathrm{L}^{-1}$ & $56.9 \pm 81.8$ & $112.1 \pm 109.6$ & $134.2 \pm 216.9$ & 0.0145 \\
\hline HOMA-IR & $4.0 \pm 6.9$ & $4.8 \pm 2.4$ & $6.3 \pm 2.8$ & 0.3899 \\
\hline
\end{tabular}

Data are expressed as $\mathrm{n}$ or mean \pm SD, unless otherwise stated. ALT: alanine aminotransferase; AST: aspartate aminotransferase; GGT: $\gamma$-glutamyl transpeptidase; HOMA-IR: homeostasis model assessment of insulin resistance.

for age, sex, metabolic syndrome and HOMA-IR (OR, 4.73, 95\% CI 1.25-17.88 and OR 4.79, 95\% CI 1.18-19.54, respectively) (supplementary table S1).

\section{Discussion}

To the best of our knowledge, this is the largest study evaluating the relationship between OSA severity and LSM. This study demonstrated that patients with severe OSA and metabolic comorbidities are at higher risk of significant liver disease (LSM $\geqslant 7.3 \mathrm{kPa}$ ) and advanced liver fibrosis (LSM $\geqslant 9.6 \mathrm{kPa}$ ) after adjustment for the main factors contributing to the development and exacerbation of NAFLD, including age, central obesity, metabolic syndrome and insulin resistance. AHI and ODI were the factors with the strongest independent association with LSM, whereas T90 was not associated with advanced liver fibrosis. While ODI reflects the frequency of oxygen desaturations, T90 reflects the severity of hypoxaemia and is less specific of OSA-related hypoxaemia [23]. These data are in accordance with previously published clinical reports and basic research suggesting the major role of intermittent hypoxia in the hepatic consequences of OSA $[3,4]$.

This study underlines the high proportion of patients at risk of significant liver lesions, especially liver fibrosis, among patients with metabolic comorbidities investigated for suspected OSA, particularly among

LSM $\geqslant 9.6 \mathrm{kPa} \quad \square$ LSM $7.3-<9.6 \mathrm{kPa} \quad \square \mathrm{LSM}<7.3 \mathrm{kPa}$

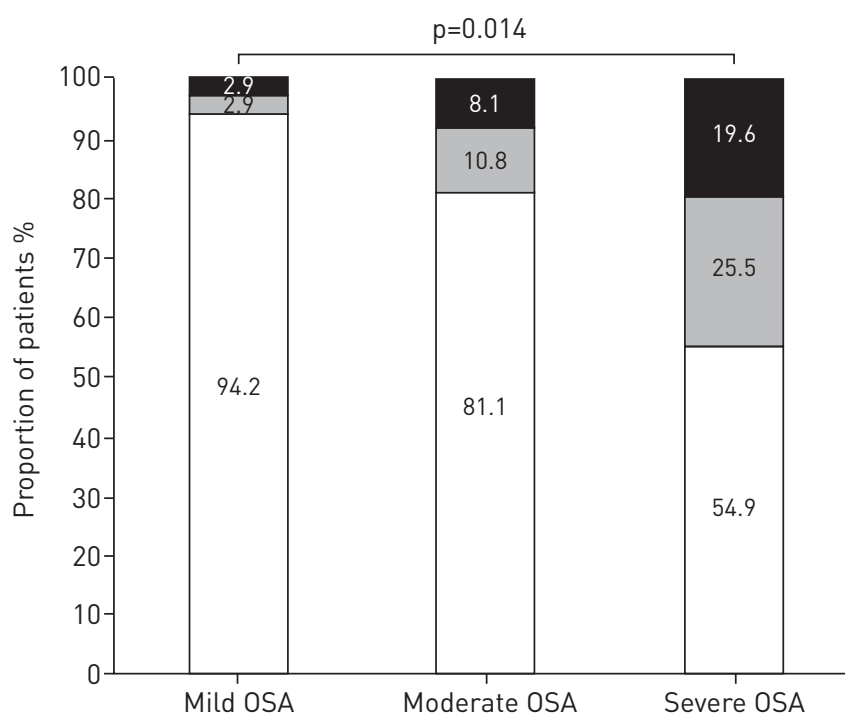

FIGURE 2 Relationship between obstructive sleep apnoea (OSA) severity and liver stiffness measurement (LSM). The following commonly used cut-offs for the apnoea-hypopnoea index were used to define categories of OSA severity: $5-<15$ events $\cdot h^{-1}$ for mild OSA, $15-<30$ events $\cdot h^{-1}$ for moderate OSA and $\geqslant 30$ events $\cdot h^{-1}$ for severe OSA. 
TABLE 3 Unadjusted and adjusted odds ratios for having a liver stiffness measurement $\geqslant 7.3 \mathrm{kPa}$ according to the severity of obstructive sleep apnoea and hypoxaemia during sleep

\begin{tabular}{|c|c|c|c|c|}
\hline & \multirow[t]{2}{*}{ Unadjusted OR $(95 \% \mathrm{CI})$} & \multicolumn{3}{|c|}{ Adjusted OR $(95 \% \mathrm{CI})^{\#}$} \\
\hline & & Model 1 & Model 2 & Model 3 \\
\hline \multicolumn{5}{|c|}{ AHI events $\cdot h^{-1}$} \\
\hline$<30$ & 1 & 1 & 1 & 1 \\
\hline$\geqslant 30$ & $5.66(2.32-13.79)$ & 6.59 (2.35-18.49) & 5.63 (2.17-14.63) & $7.17(2.51-20.50)$ \\
\hline \multicolumn{5}{|c|}{ ODI events $\mathrm{h}^{-1}$} \\
\hline$<19$ & 1 & 1 & 1 & 1 \\
\hline$\geqslant 19$ & $4.86(2.01-11.78)$ & $6.62(2.17-20.22)$ & $4.79(1.80-12.74)$ & $6.23(2.11-18.42)$ \\
\hline \multicolumn{5}{|l|}{$T 90 \%$} \\
\hline$<2$ & 1 & 1 & 1 & 1 \\
\hline$\geqslant 2$ & $4.46(1.75-11.38)$ & $4.51(1.53-13.25)$ & 3.75 (1.39-10.12) & $4.29(1.50-12.23)$ \\
\hline
\end{tabular}

AHI: apnoea-hypopnoea index; ODI: oxygen desaturation index; T90: sleep time with oxygen saturation $<90 \%$. \#: model 1: adjusted for age, sex, waist circumference, diabetes and hypertension; model 2 : adjusted for age, sex and metabolic syndrome; model 3: adjusted for age, sex, metabolic syndrome and homeostasis model assessment of insulin resistance.

those with severe OSA. This finding is consistent with histological data from previous studies performed in obese patients undergoing bariatric surgery showing that almost $50 \%$ of patients with severe OSA present significant fibrosis (stage F2 or greater) $[4,24]$. These findings suggest that, apart from extremely obese patients, a significant proportion of severe OSA patients should be referred to hepatologists for specific management.

Identification and quantification of fibrosis is a key issue in patients with NAFLD because fibrosis stage, but no other histological feature of steatohepatitis, is independently associated with long-term outcomes [5]. However, the independent association between OSA severity and liver fibrosis remains the subject of debate. Biopsy-based studies evaluating liver fibrosis have reported conflicting results, as some demonstrated an independent association between liver fibrosis and markers of nocturnal hypoxaemia [25], while others failed to demonstrate an independent association after adjustment for major NAFLD risk factors $[26,27]$. These studies were conducted in the particular population of morbidly obese patients undergoing intra-operative needle liver biopsy during bariatric surgery, thereby limiting generalisation of the findings and presumably contributing to the relative statistical weakness of the association observed, as up to $91 \%$ of the patients included were female and less prone to develop android obesity and its metabolic and respiratory consequences $[4,28]$. Two other studies investigated the association between OSA and the various components of NAFLD in larger cohorts of patients investigated by PSG and blood tests for evaluation of NAFLD lesions. These studies demonstrated an independent association between OSA severity and steatosis, but the association with liver fibrosis markers was no longer significant after adjustment for major risk factors, including age and waist circumference [29, 30]. The algorithms used to predict liver fibrosis included age, BMI, sex and serum transaminase levels. As these various parameters have been shown to correlate with or be impacted by OSA, further adjustments in multivariate analysis would be of limited value.

This limitation can be overcome by evaluating liver lesions with LSM, a physical measurement based on elastography. To the best of our knowledge, only one study has evaluated LSM in patients with OSA, and found an independent correlation between the severity of intermittent hypoxia and increased fibrosis as determined by LSM [31]. This study presented a major limitation due to the mix of two different cohorts of NAFLD and OSA patients, and a very small sample of patients in which both PSG and LSM data were available (PSG was performed in only 31 of the 123 patients included in the analysis).

We acknowledge that liver biopsy was not available in our study. Although it remains the reference to evaluate liver lesions in NAFLD, this procedure is seldom used in clinical practice because of its invasiveness [6]. In addition, liver biopsy is not a perfect gold standard because of sampling bias and suboptimal interobserver reproducibility [32, 33]. Blood tests and elastography devices have been developed to facilitate the diagnosis of liver fibrosis and these noninvasive tests are now widely used in clinical practice. A recent meta-analysis has shown that FibroScan LSM $\geqslant 9.6 \mathrm{kPa}$ has excellent sensitivity and negative predictive value for the diagnosis of advanced liver fibrosis in NAFLD, but only a moderate $68 \%$ positive predictive value [18]. We therefore cannot affirm that all patients with LSM $\geqslant 9.6 \mathrm{kPa}$ in our study had advanced liver fibrosis. However, blood fibrosis tests and FibroScan are highly correlated with 
liver fibrosis, and have been recently shown to be themselves prognostic markers able to identify NAFLD patients with impaired outcome [10,34]. This represents a strong argument for the use of these tests in clinical practice to assess NAFLD severity and identify the subgroup of patients justifying referral to specialised hepatologists for specific evaluation and management [35]. For the same reason, noninvasive tests of liver fibrosis are relevant tools in clinical studies such as the present work to evaluate the relationships between NAFLD severity and the different complications of obesity and metabolic syndrome.

Another potential limitation of the present study concerns the selection of the sample. We included patients presenting with a clinical suspicion of OSA and at least one criterion for metabolic syndrome. Our findings are in accordance with previous experimental and clinical studies demonstrating that OSA and intermittent hypoxia exacerbate the metabolic dysfunction of obesity [36]. However, the present findings should not be generalised to all OSA patients and further studies are required to investigate the impact of OSA severity on LSM in patients without metabolic comorbidities.

Finally, because of the cross-sectional design of this study, it cannot be determined whether OSA preceded onset of increased liver stiffness. Investigations into the causal link between OSA and liver damage will require prospective studies. In a recent randomised trial, 6-12 weeks of nasal continuous positive airway pressure, i.e. the reference treatment for moderate-to-severe OSA, did not improve blood markers of liver injury [37]. Further studies are required to determine whether long-term OSA therapy, by abolishing nocturnal oxygen desaturation, can help to prevent progression of liver stiffness.

In conclusion, severe OSA in patients with metabolic comorbidities is independently associated with increased liver stiffness, which may predispose to a higher risk of significant liver disease and poorer prognosis.

Acknowledgements: The members of the METABOL Group are as follows: Dépt de Pneumologie, CHU, Angers, France: Nicole Meslier, Pascaline Priou; Service d'Hépato-Gastroentérologie, CHU, Angers, France: Frédéric Oberti, Isabelle Fouchard-Hubert, Adrien Lannes, Sandra Girres; Dépt d'Endocrinologie, Diabétologie et Nutrition, CHU, Angers, France: Ingrid Allix; Dépt de Médecine du Sport et Explorations Fonctionnelles Vasculaires, CHU, Angers, France: Georges Leftheriotis; INSERM UMR 1063 "SOPAM", Univeristé d'Angers, Angers, France: Ramaroson Andriantsitohaina, Carmen Martinez, Soazig Le Lay, Raffaella Soleti, Luisa Vergori; Dépt de la Recherche Clinique et Innovation, $\mathrm{CHU}$, Angers, France: Jean-Marie Chrétien; Centre de Resource Biologique, CHU, Angers, France: Odile Blanchet, Belaid Sekour; Laboratoire HIFIH, EA3859, Université d’Angers, France: Gilles Hunault.

Conflict of interest: J. Boursier and P. Calès report personal fees from Echosens, a company that has a FibroMeter licence from Université d'Angers, during the conduct of the study.

Support statement: This study was funded by Université d'Angers. Funding information for this article has been deposited with the Crossref Funder Registry.

\section{References}

Ogden CL, Carroll MD, Flegal KM. Prevalence of obesity in the United States. JAMA 2014; 312: 189-190

Kopelman PG. Obesity as a medical problem. Nature 2000; 404: 635-643.

Aron-Wisnewsky J, Clement K, Pépin J-L. Nonalcoholic fatty liver disease and obstructive sleep apnea. Metabolism 2016; 65: 1124-1135.

4 Aron-Wisnewsky J, Minville C, Tordjman J, et al. Chronic intermittent hypoxia is a major trigger for non-alcoholic fatty liver disease in morbid obese. J Hepatol 2012; 56: 225-233.

5 Dulai PS, Singh S, Patel J, et al. Increased risk of mortality by fibrosis stage in nonalcoholic fatty liver disease: systematic review and meta-analysis. Hepatology 2017; 65: 1557-1565.

Bravo AA, Sheth SG, Chopra S. Liver biopsy. N Engl J Med 2001; 344: 495-500.

7 Asrani SK. Incorporation of noninvasive measures of liver fibrosis into clinical practice: diagnosis and prognosis Clin Gastroenterol Hepatol 2015; 13: 2190-2204.

8 Machado MV, Cortez-Pinto H. Non-invasive diagnosis of non-alcoholic fatty liver disease. A critical appraisal. J Hepatol 2013; 58: 1007-1019.

9 Castera L, Forns X, Alberti A. Non-invasive evaluation of liver fibrosis using transient elastography. $J$ Hepatol 2008; 48: 835-847.

10 Boursier J, Vergniol J, Guillet A, et al. Diagnostic accuracy and prognostic significance of blood fibrosis tests and liver stiffness measurement by FibroScan in non-alcoholic fatty liver disease. J Hepatol 2016; 65: 570-578.

11 Expert Panel on Detection, Evaluation, and Treatment of High Blood Cholesterol in Adults. Executive Summary of the Third Report of the National Cholesterol Education Program (NCEP) Expert Panel on Detection, Evaluation, and Treatment of High Blood Cholesterol in Adults (Adult Treatment Panel III). JAMA 2001; 285: 2486-2497.

12 Monseu M, Dubois S, Boursier J, et al. Osteoprotegerin levels are associated with liver fat and liver markers in dysmetabolic adults. Diabetes Metab 2016; 42: 364-367.

13 Netzer NC, Stoohs RA, Netzer CM, et al. Using the Berlin Questionnaire to identify patients at risk for the sleep apnea syndrome. Ann Intern Med 1999; 131: 485-491.

14 Gagnadoux F, Le Vaillant M, Goupil F, et al. Depressive symptoms before and after long-term CPAP therapy in patients with sleep apnea. Chest 2014; 145: 1025-1031.

15 Matthews DR, Hosker JP, Rudenski AS, et al. Homeostasis model assessment: insulin resistance and beta-cell function from fasting plasma glucose and insulin concentrations in man. Diabetologia 1985; 28: 412-419. 

liver disease: a meta-analysis of diagnostic accuracy. J Hepatol 2011; 54: 650-659.

17 Wong VW-S, Vergniol J, Wong GL-H, et al. Diagnosis of fibrosis and cirrhosis using liver stiffness measurement in nonalcoholic fatty liver disease. Hepatology 2010; 51: 454-462.

18 Xiao G, Zhu S, Xiao X, et al. Comparison of laboratory tests, ultrasound, or magnetic resonance elastography to detect fibrosis in patients with nonalcoholic fatty liver disease: a meta-analysis. Hepatology 2017; 66: 1486-1501.

19 Boursier J, Zarski J-P, de Ledinghen V, et al. Determination of reliability criteria for liver stiffness evaluation by transient elastography. Hepatology 2013; 57: 1182-1191.

20 Schwabl P, Bota S, Salzl P, et al. New reliability criteria for transient elastography increase the number of accurate measurements for screening of cirrhosis and portal hypertension. Liver Int 2015; 35: 381-390.

21 Gagnadoux F, Pépin J-L, Vielle B, et al. Impact of mandibular advancement therapy on endothelial function in severe obstructive sleep apnea. Am J Respir Crit Care Med 2017; 195: 1244-1252.

22 Berry RB, Budhiraja R, Gottlieb DJ, et al. Rules for scoring respiratory events in sleep: update of the 2007 AASM Manual for the Scoring of Sleep and Associated Events. Deliberations of the Sleep Apnea Definitions Task Force of the American Academy of Sleep Medicine. J Clin Sleep Med 2012; 8: 597-619.

23 Kendzerska T, Gershon AS, Hawker G, et al. Obstructive sleep apnea and risk of cardiovascular events and all-cause mortality: a decade-long historical cohort study. PLoS Med 2014; 11: e1001599.

24 Mesarwi OA, Shin M-K, Drager LF, et al. Lysyl oxidase as a serum biomarker of liver fibrosis in patients with severe obesity and obstructive sleep apnea. Sleep 2015; 38: 1583-1591.

25 Sookoian S, Pirola CJ. Obstructive sleep apnea is associated with fatty liver and abnormal liver enzymes: a meta-analysis. Obes Surg 2013; 23: 1815-1825.

26 Jouët P, Sabaté J-M, Maillard D, et al. Relationship between obstructive sleep apnea and liver abnormalities in morbidly obese patients: a prospective study. Obes Surg 2007; 17: 478-485.

27 Kallwitz ER, Herdegen J, Madura J, et al. Liver enzymes and histology in obese patients with obstructive sleep apnea. J Clin Gastroenterol 2007; 41: 918-921.

28 Musso G, Cassader M, Olivetti C, et al. Association of obstructive sleep apnoea with the presence and severity of non-alcoholic fatty liver disease. A systematic review and meta-analysis. Obes Rev 2013; 14: 417-431.

29 Minville C, Hilleret M-N, Tamisier R, et al. Nonalcoholic fatty liver disease, nocturnal hypoxia, and endothelial function in patients with sleep apnea. Chest 2014; 145: 525-533.

30 Trzepizur W, Boursier J, Mansour Y, et al. Association between severity of obstructive sleep apnea and blood markers of liver injury. Clin Gastroenterol Hepatol 2016; 14: 1657-1661.

31 Agrawal S, Duseja A, Aggarwal A, et al. Obstructive sleep apnea is an important predictor of hepatic fibrosis in patients with nonalcoholic fatty liver disease in a tertiary care center. Hepatol Int 2015; 9: 283-291.

32 Bedossa P, Dargère D, Paradis V. Sampling variability of liver fibrosis in chronic hepatitis C. Hepatology 2003; 38: 1449-1457.

33 Rousselet M-C, Michalak S, Dupré F, et al. Sources of variability in histological scoring of chronic viral hepatitis. Hepatology 2005; 41: 257-264.

34 Angulo P, Bugianesi E, Bjornsson ES, et al. Simple noninvasive systems predict long-term outcomes of patients with nonalcoholic fatty liver disease. Gastroenterology 2013; 145: 782-789.

35 Boursier J, de Ledinghen V, Leroy V, et al. A stepwise algorithm using an at-a-glance first-line test for the non-invasive diagnosis of advanced liver fibrosis and cirrhosis. J Hepatol 2017; 66: 1158-1165

36 Drager LF, Togeiro SM, Polotsky VY, et al. Obstructive sleep apnea: a cardiometabolic risk in obesity and the metabolic syndrome. J Am Coll Cardiol 2013; 62: 569-576.

37 Jullian-Desayes I, Tamisier R, Zarski J-P, et al. Impact of effective versus sham continuous positive airway pressure on liver injury in obstructive sleep apnoea: data from randomized trials. Respirology 2016; 21: 378-385. 